\title{
ESTUDO DA APRENDIZAGEM MEDIADA POR COMPUTADOR: AS CONTRIBUIÇõES DA MODELAGEM MOLECULAR PARA O ENSINO DE QUÍMICA
}

Adriana de Farias Ramos - IFRS/Campus POA (adrfram@gmail.com)

Agostinho Serrano de Andrade Neto - ULBRA/RS (asdraden@gmail.com)

\section{RESUMO}

0 objetivo deste trabalho é mostrar evidências da aquisição de novas representações e de novos drivers por parte de estudantes de química quando utilizam software de modelagem molecular. Neste trabalho, utilizamos referenciais inéditos na educação química: o referencial teórico da Teoria da Mediação Cognitiva em Rede (TMC) e a metodologia de combinar análise de discurso e análise gestual. Os registros usados são os testes escritos (pré e pós) e os vídeos gravados durante entrevista semiestruturada conforme protocolo Report Aloud. Desses vídeos, extraímos os gestos (discurso não verbal), que foram avaliados por análise gestual, e o discurso verbal, que foi avaliado por análise de discurso. Ao final deste recorte, conseguimos mostrar, tanto nos gestos quanto no discurso verbal e na escrita, os indícios da presença dessas novas representações e dos novos drivers que foram construídos a partir da mediação por computador. Por isso, os estudantes demonstraram ter criado uma competência representacional que permitiu a integração de conceitos químicos.

Palavras- chave: ensino de química, modelagem molecular, cognição extracerebral, aprendizagem mediada por computador.

\section{STUDY OF COMPUTER-MEDIATED LEARNING: CONTRIBUTIONS OF MOLECULAR MODELING FOR CHEMISTRY EDUCATION}

\begin{abstract}
The aim of this work is to show evidence of the acquisition of new representations and drivers by chemistry students when they use molecular modeling software. In this work, we use novel referentials for chemistry education: the theoretical referential of the Networks Cognitive Mediation theory (TMC) and the methodology of combining discourse and gesture analysis. The data used were the written tests (pre and post) and videos recorded during an interview conducted according to protocol Aloud Report. From these videos, we extract the gestures (nonverbal discourse), which were evaluated by gestural analysis, and the verbal discourse, which were evaluated by analysis of discourse. At the end of this outline research, we show both, in gesture as well as in speech and writing, evidence of the presence of new representations and drivers, which were built from the computermediated. On that account, students demonstrated have constructed a representational competence that allowed the integration of chemical concepts.
\end{abstract}

Keywords: chemistry education, molecular modeling, extracerebral cognition, computer-mediated learning. 


\title{
1. INTRODUÇÃO
}

A comunidade de pesquisadores em ensino de ciências tem debatido por décadas formas de proporcionar aos estudantes uma melhor aprendizagem de conteúdos da área. Contudo, nas salas de aula ainda prevalece o modelo de transmissão (BRITO; LOPES, 2011). Santos e Greca (2005) revelaram que o panorama do ensino de química é preocupante porque:

\begin{abstract}
Muitos estudantes têm dificuldade em compreender as representações em química. As compreensões microscópica e simbólica são especialmente difíceis porque são invisíveis e abstratas e o pensamento dos alunos é construído sobre a informação sensorial. Além disso, os estudantes não estabelecem relações apropriadas entre o nível macro e o microscópico e ainda, muitos que tenham conhecimento conceitual e habilidade de visualizar, são incapazes de transladar de uma dada representação química a outra. (SANTOS; GRECA, 2005, p. 1).
\end{abstract}

As habilidades espaciais são importantes para a compreensão de vários conceitos das ciências, incluindo a matemática (BERNARDES; NOTARE, 2013). Em relação à química, a adoção de estratégias diversas para o desenvolvimento das habilidades visuoespaciais dos estudantes contribui para a construção de uma competência representacional dos modelos tridimensionais das moléculas, levando à facilidade de compreensão de outras propriedades dependentes da geometria molecular. Essa habilidade de visualização é definida como " $a$ habilidade necessária para transitar entre os níveis representacionais e é chamada de habilidade visuoespacial - derivada do conceito visualização espacial" (RAUPP et al., 2009). Seu desenvolvimento proporciona o avanço para a consolidação de imagens mentais de rotação intramolecular e intermolecular em 3D. 0 conhecimento das imagens mentais produzidas pelos estudantes é peça-chave para definição de novas estratégias didáticas (BASGALUPE; SERRANO, 2013).

A criação e a consolidação de imagens mentais de rotação intramolecular e intermolecular das moléculas em 3D depende da capacidade de construção de modelos. A importância da construção de modelos nas ciências já é consagrada (FERREIRA; JUSTI, 2008). Nesse contexto, a modelagem tem sido cada vez mais usada para atividades didáticas (RODRIGUES, 2012) e pode ser considerada um processo de construção e reformulação de modelos. A modelagem em química, como um processo de construção e reformulação de modelos, tem um uso bem amplo e abrangente, com diversas e distintas metodologias, incluindo a modelagem molecular, que se realiza com o uso de softwares específicos. Neste trabalho, utilizamos essa modelagem compreendendo-a como "a aplicação de modelos teóricos para representar e manipular a estrutura de moléculas, estudar reações químicas e estabelecer relações entre a estrutura e propriedades da matéria" (SANTOS, 2001).

Isso posto, achamos importante fazer uma distinção entre os softwares de visualização e os de modelagem molecular. Os primeiros comportam atividades mais simples, de construção e visualização de moléculas em 3D. Por sua vez, os V. $12 \mathrm{~N}^{\mathrm{o}} 2$, julho, 2014 
softwares de modelagem molecular possuem uma gama de possibilidades de cálculos e tarefas muito mais complexas, oportunizando a realização de inúmeras atividades que podem envolver processos de pensamento mais abstrusos, como, por exemplo, a predição do comportamento de um determinado sistema químico. Sendo assim, o emprego de softwares de modelagem molecular vai além da visualização e se constitui em poderosa ferramenta de processamento externo de informações. Sem eles não seria possível a abordagem de conteúdos específicos que envolvem cálculos mais intrincados, além de propiciar aos estudantes o desenvolvimento de habilidades de pensamento mais complexas (KABERMAN; DORI, 2007).

O objetivo deste trabalho é mostrar indícios da existência de novas representações e de novos drivers na estrutura cognitiva dos estudantes após o uso de softwares de modelagem molecular. Para tanto, partimos da seguinte pergunta de pesquisa: é possível se observar a gênese de representações e drivers na estrutura cognitiva de estudantes após o uso de software de modelagem molecular? Diante disso, realizamos um recorte de nossa pesquisa de doutoramento, a qual busca compreender quais modificações ocorrem na estrutura cognitiva dos alunos quando utilizam esses softwares para resolver problemas químicos. A Figura 1 mostra o resumo do referencial teóricoepistemológico estruturado para sustentar os resultados da pesquisa.

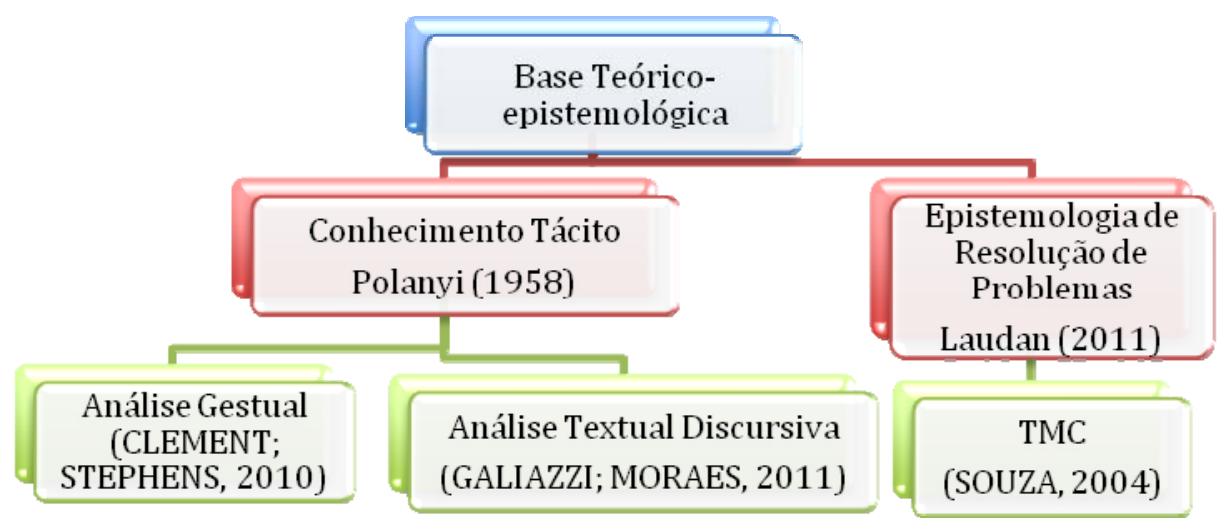

Figura 1: Resumo do referencial teórico-epistemológico adotado.

Utilizamos como base teórica um referencial inédito para a área da educação química, a Teoria da Medicação Cognitiva, que se associa muito bem à epistemologia de resolução de problemas de Larry Laudan, usada para embasar os testes escritos. A metodologia da pesquisa, igualmente inédita para a área, foi construída a partir da articulação da epistemologia do conhecimento tácito de Polanyi (POLANYI, 1958) com a análise gestual (MONAGHAN; CLEMENT, 1999; CLEMENT; STEPHENS, 2010), para desvendarmos o discurso não verbal, e a análise textual discursiva (GALIAZZI; MORAES, 2011), para avaliarmos o discurso verbal.

\section{REFERENCIAIL TEÓRICO}

Nossa pesquisa partiu da concepção de que a ciência tem como característica ser uma atividade de resolução de problemas (LAUDAN, 2011) e V. $12 \mathrm{~N}^{\mathrm{o}} 2$, julho, 2014 
utilizou como arcabouço teórico a Teoria da Mediação Cognitiva em Rede - TMC (SOUZA, 2004; SOUZA et al., 2012), que procurou explicar o processamento da informação pelo cérebro, propiciando uma abordagem ampla para a cognição humana.

A TMC foi fundamentada em cinco premissas relativas à cognição humana e ao processamento de dados:

\begin{abstract}
(...) 1) A espécie humana tem como maior vantagem evolutiva a capacidade de gerar, armazenar, recuperar, manipular e aplicar o conhecimento de várias maneiras; 2) Cognição humana é efetivamente o resultado de algum tipo de processamento de informação; 3) Sozinho, o cérebro humano constitui um finito e, em última instância, insatisfatório, recurso de processamento de informação; 4) Praticamente qualquer sistema físico organizado é capaz de executar operações lógicas em algum grau; 5) Seres humanos complementam o processamento da informação cerebral por interação com os sistemas físicos externos organizados. (SOUZA et al., 2012, p.2, tradução nossa).
\end{abstract}

A cognição humana é o resultado de processamento de informações, e uma parte importante dessa atividade é realizada fora do cérebro, visto ser ele limitado para elaborar todas as informações disponíveis. Nesse sentido, utilizamos o processamento externo por meio da interação com estruturas do ambiente para aumentar a capacidade de processamento de informações.

Por exemplo, quando usamos um computador para processar informações, ou mesmo realizar um cálculo mais complexo, o estamos utilizando como um mecanismo externo de mediação. Para tanto, precisamos construir alguns mecanismos internos em nossa estrutura cognitiva que nos possibilite manusear esse computador e compreender não somente seu funcionamento, mas também as informações que ele está nos oferecendo. São os mecanismos internos que tornam possível o emprego dos mecanismos externos, e esses mecanismos internos são chamados pelo autor da TMC de "drivers", os quais se desenvolvem a partir da interação entre o indivíduo e o mecanismo externo de processamento de informações por meio do método processo piagetiano de equilibração.

Isso significa que, para garantir o processo de mediação cognitiva com um mecanismo externo, nosso cérebro cria novos drivers que o dotam de competências específicas para se comunicar com esse mecanismo. Com isso, adquire um ganho de processamento de informações que se mantém mesmo que a conexão com o mecanismo externo seja interrompida. E esse ganho é considerado aquisição de conhecimentos pelo autor da TMC .

Talvez a premissa mais interessante da TMC para nossa pesquisa seja que 0 cérebro busca instrumentos externos capazes de completar suas limitações naturais, como no desenvolvimento histórico da química computacional. Esse processo de mediação resulta na aquisição de uma competência representacional advinda da construção de representações e drivers, ampliando sobremaneira a capacidade de processar informações do cérebro. Diante disso, optamos neste artigo por mostrar os indícios da presença dessas representações e desses drivers provenientes do processo de mediação cognitiva extracerebral. Em trabalho 
recente, Rocha e Serrano (2013) mostraram que a TMC também se encaixa muito bem para explicar as modificações cognitivas resultantes do uso de simulações na área da física.

\section{DELINEAMENTOS METODOLÓGICOS}

A metodologia que sustenta os resultados deste recorte da nossa pesquisa é do tipo microetnográfica (MOREIRA, 2002) e conta com as seguintes etapas metodológicas: pré-teste teórico; modelagem molecular utilizando o software Spartan; pós-teste teórico com as mesmas questões do pré-teste; entrevista semiestruturada baseada no protocolo Report Aloud. Essa metodologia foi construída após a realização de dois experimentos piloto e implementada em um experimento definitivo realizado com cinco estudantes de graduação em química da Universidade Luterana do Brasil, num curso de extensão de 15 horas.

Os dados para análise consistem em: testes escritos (pré e pós), 5 horas de vídeo das entrevistas e aproximadamente 100 páginas de transcrições das mesmas com a identificação e explicação de todos os gestos descritivos realizados pelos estudantes quando narravam os processos de raciocínio para a resolução das questões dos testes escritos. A Figura 2, a seguir, mostra como foram organizados os registros para análise.

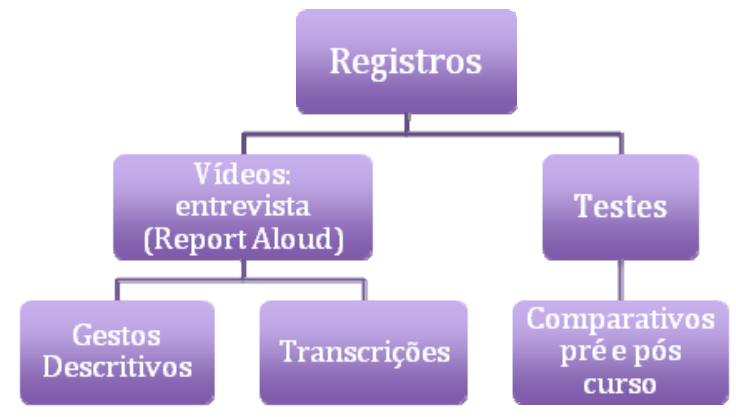

Figura 2: Organização de registros para análise.

As entrevistas foram conduzidas segundo o protocolo Report Aloud, adaptado por nós do protocolo Think Aloud (VAN-SOMEREN et al., 1994). 0 protocolo Report Aloud difere do protocolo Think Aloud apenas no momento em que o pesquisador busca compreender o processo de raciocínio dos estudantes na resolução de problemas. Enquanto o método Think Aloud explora os processos de pensamento dos alunos no ato da resolução do problema, interferindo nesse processo, o método Report Aloud o faz após o estudante ter resolvido o problema. Dessa forma, não há a interferência do pesquisador no próprio processo de raciocínio do estudante e o resgate fidedigno desse raciocínio é possível, a depender da habilidade do pesquisador em explorar as representações e os drivers existentes na estrutura cognitiva dos estudantes, identificando quais já existiam (conhecimento tácito) e quais foram adquiridas ao longo do curso.

Em nossa pesquisa, o conhecimento tácito é a base fundamental de análise, junto com o conhecimento explícito do discurso do estudante durante as entrevistas. Ao se deparar com uma atividade de resolução de problemas, ele irá evocar um conjunto de conhecimentos prévios e, conforme já argumentamos, boa parte dele é tácito. Naturalmente é impossível a análise do conhecimento tácito se este não for transformado em explícito. Por isso, resolvemos focar de modo V. $12 \mathrm{~N}^{\mathrm{o}} 2$, julho, 2014 
especial no conhecimento tácito que se apresenta como imagens mentais, pois entendemos que, dessa maneira, os conhecimentos tácitos são transformados em explícitos por elementos específicos, diretos e indiretos, do discurso do estudante, sobretudo sob a forma de gestos descritivos.

Para analisar os gestos descritivos utilizamos como base a linha de trabalho que revela o conhecimento implícito inerente à visualização interna, mediante externalização por análise gestual (MONAGHAN; CLEMENT, 1999). A partir dessa metodologia, é possível identificar padrões de gestos e relacioná-los com os conhecimentos implícitos existentes na estrutura cognitiva dos estudantes (CLEMENT; STEPHENS, 2010). O que Clement e Stephens chamam de imagens mentais e simulações mentais interpretamos aqui, no contexto de nosso referencial teórico, como representações e drivers.

\section{RESULTADOS}

Com este recorte, nos propomos a mostrar evidências - na fala, nos gestos e nos testes teóricos - da aquisição de novas representações e de novos drivers provenientes da mediação com o computador, em especial com a modelagem molecular. Como exemplo de aquisição de representações e drivers, trazemos o caso do estudante P. Em relação à análise conformacional, esse estudante afirmou na entrevista que conseguia visualizar mentalmente uma rotação intramolecular 3D e que essa imagem mental não existia antes do curso.

Trata-se de uma capacidade de usar a mente para rotacionar partes da molécula, adquirida sob a forma de representações e drivers, com o uso do software. A Figura 3, a seguir, mostra o estudante realizando uma sequência de gestos descritivos quando estava explicando como enxergava mentalmente a molécula do dicloroetano girando.

(a)

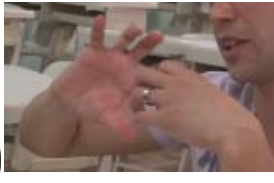

(b)

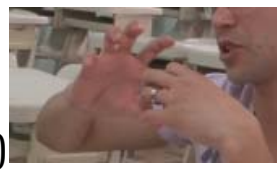

(c)

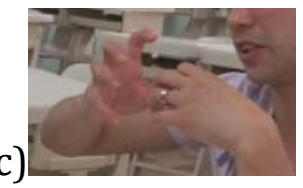

(d)

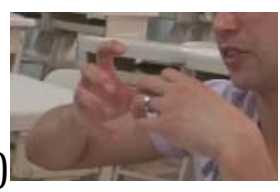

(a)

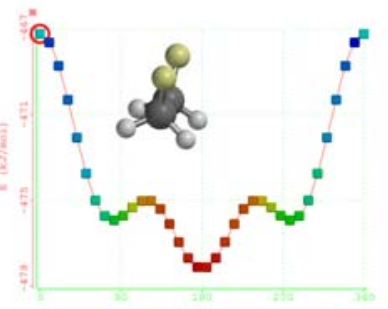

(c)

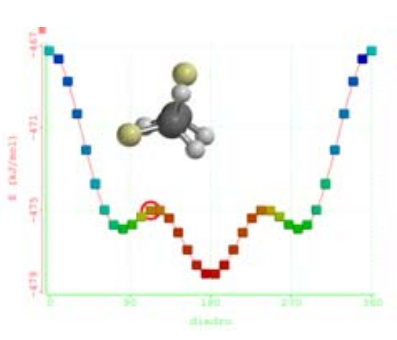

(b)

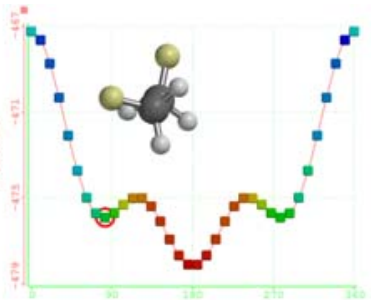

(d)

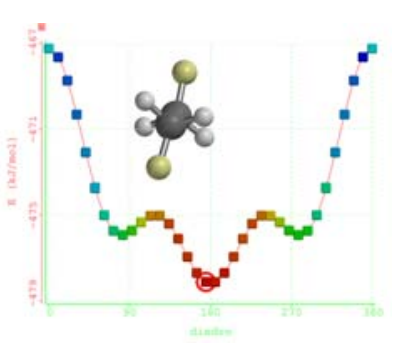

Figura 3: Estudante P descrevendo em gestos uma torção intramolecular com a respectiva simulação de modelagem molecular.

V. $12 \mathrm{~N}^{\mathrm{o}} 2$, julho, 2014 
Nessa sequência de gestos, o estudante coloca ambas as mãos de frente uma para a outra. Então, ele fixa a mão esquerda em forma de concha e gira a mão direita, também em forma de concha, num eixo de rotação perpendicular ao plano formado pela mão esquerda. Para tanto, precisou criar representações e drivers a partir da mediação com a simulação de modelagem molecular que realizou.

O formato das mãos do estudante seguiu um padrão universal identificado nos gestos de todos os estudantes: a mão em forma de concha para representar átomos isolados ou mesmo partes da molécula. Essas representações e drivers foram desvendadas pelos gestos, combinados com a fala, do estudante, que, em dado momento da entrevista, afirmou:

\begin{abstract}
Eu imagino o carbono. Pra mim, eu imagino um lado fixo e o outro carbono, ele está girando e enquanto ele vai girando as outras extremidades (as ligadas neste último carbono) vão girando e acompanhando ele. Então fica um lado o carbono com o cloro e o hidrogênio e o outro lado sempre girando. (...) eu não imaginava assim antes. (Estudante P).
\end{abstract}

A imagem acima mostra que o estudante internalizou o conteúdo da modelagem molecular, que aqui pode ser considerada um mecanismo externo de processamento do informações. 0 estudante, ao tomar contato com a modelagem molecular que ele mesmo construiu em uma atividade proposta no curso de extensão, desenvolve novos drivers a partir de um processo de equilibração piagetiana. Esses drivers permanecem na estrutura cognitiva do estudante, mesmo depois de "desconectado" do mecanismo de processamento extracerebral. Esses novos drivers são identificados a partir da descrição do próprio aluno, demonstrada na Figura 3. Além disso, ele afirma que não tinha construído anteriormente essa representação. Então, identificamos aqui a aquisição de drivers que permitem ao estudante ter uma visão dinâmica de um comportamento intramolecular, adquirida pela mediação com o software de modelagem molecular.

Soma-se a isso a constatação da mudança no padrão de representação do estudante $\mathrm{P}$ em relação à construção de geometrias moleculares quando comparamos as respostas de seus pré e pós-testes, conforme explicitado na Figura 4 a seguir.

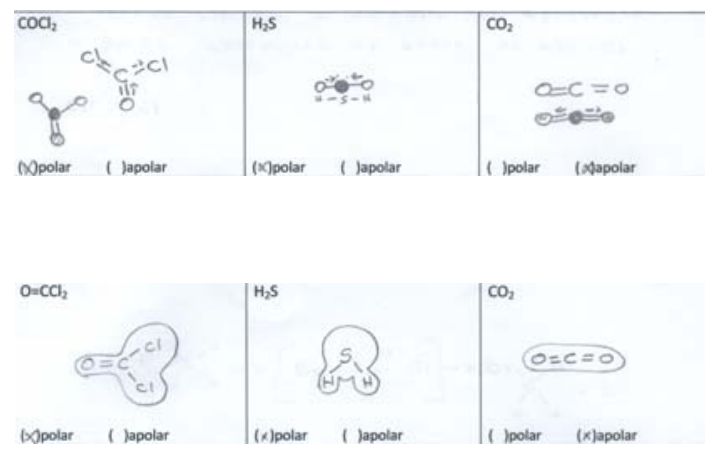

(A)

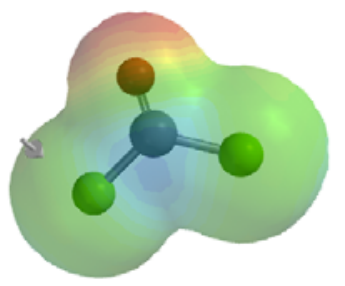

(C)

(B)

Figura 4: Comparativo entre pré e pós-testes do estudante $\mathrm{P}$ em relação à questão envolvendo geometria molecular. (A) moléculas no pré-teste; (B) moléculas no pós-teste; (C) moléculas no software Spartan.

V. $12 \mathrm{~N}^{\mathrm{o}} 2$, julho, 2014 
Fica claro que houve modificação na forma de representar as moléculas, se compararmos o pré-teste com o pós-teste. Nesta questão, os alunos tinham que montar as geometrias das moléculas, indicando se as mesmas eram polares ou apolares. Ao longo do curso, fizemos alguns exercícios em que eles eram convidados a montar as moléculas no software, otimizar as geometrias e plotar o mapa de potencial eletrostático para perceber se eram polares ou apolares. A principal modificação que se percebe é a qualidade conceitual aliada a melhor competência representacional do estudante, que, além de ter construído as moléculas com a geometria adequada, também colocou o contorno do mapa de potencial eletrostático das moléculas.

\section{CONSIDERAÇÕES}

Esse recorte de nossa pesquisa nos permite dizer, de uma maneira geral, que o uso do software de modelagem molecular permitiu ao estudante a construção de novas representações e novos drivers, que se mantiveram presentes. Os dados que apresentamos nesse recorte mostram os indícios da presença deles, tanto na fala quanto nos gestos descritivos e no comparativo dos testes (pré e pós). Também percebemos, como resultado de pesquisa, que a utilização desses softwares a partir de uma estratégia didática embasada na resolução de problemas: a) auxiliou na consolidação das habilidades visuoespaciais de alguns alunos; b) propiciou aos estudantes adquirirem uma visão mais dinâmica dos processos de transformação das moléculas e, por fim, c) deu-lhes a oportunidade de construir uma visão mais integrada de conceitos que, via de regra, são tratados de forma distinta.

Pudemos mostrar indícios de internalização de conteúdos que foram mediados por computador, por meio de um software de modelagem molecular. Utilizamos a teoria da mediação cognitiva para tentar explicar de que forma ocorre a aquisição de conhecimentos a partir dessa mediação. Novos drivers são desenvolvidos para permitir esse processo de mediação e, com isso, a estrutura cognitiva do estudante vai se modificando. Para a TMC, a aquisição de conhecimentos ocorre quando novos drivers são criados, a partir de um processo piagetiano de equilibração.

A simples existência desses novos drivers dota os estudantes de ferramentas cognitivas que aumentam certas competências e o raciocínio. Esse ganho cognitivo foi evidenciado neste recorte de pesquisa na medida em que percebemos que o estudante externalizou, por gestos e explicações na fala, um conjunto de conhecimentos que foram adquiridos com o manuseio do programa. Ao analisar-lhes as respostas pelos gestos produzidos, podemos perceber que há um conhecimento implícito inerente à manipulação computacional das moléculas quando se utiliza o software.

Um caminho interessante que este estudo aponta é delinear, mesmo de forma inicial, as premissas para que os estudantes possam realizar tarefas mais complexas, que requeiram habilidades de pensamento de alta ordem como, por exemplo, realizar um raciocínio de modelagem molecular. Apontamos a articulação das três seguintes premissas: consolidação de habilidades visuoespaciais; visão dinâmica das transformações; integração de conceitos.

V. $12 \mathrm{~N}^{\mathrm{o}} 2$, julho, 2014 
A importância da habilidade visuoespacial já foi fartamente justificada neste trabalho. Ela é o ponto de partida do estudante, pois permite que ele inicie a formação de imagens mentais, produzidas pela formação de drivers específicos. A visão dinâmica das transformações oportuniza-lhe o início de um raciocínio integrador, pois ele começa a perceber que a natureza atômico- molecular não é estática e que há um equilíbrio dinâmico presente. Por fim, a integração de conceitos se estabelece à proporção que as duas primeiras premissas se consolidam na estrutura cognitiva do estudante. A partir disso, o estudante pode começar a perceber que existe uma relação estreita entre estrutura da matéria, suas propriedades e energia. Com a orientação adequada, ele tem condições de começar a construir o conceito integralizador de energia e pensar em termos de modelagem molecular, desenvolvendo habilidades de pensamento de alta ordem.

\section{REFERÊNCIAS BIBLIOGRÁFICAS}

ARAUJO, I. S.; VEIT, E. A.; MOREIRA, M. A. Modelos Computacionais no Ensinoaprendizagem de Física: um referencial de trabalo. Ivestigações em Ensino de Ciências, v. 17, n. 2, p. 341-366, 2012.

BASGALUPE, L. P. M.; SERRANO, A. Representações Mentais de Concepções Espontâneas dos Estudantes após Utilização de Softwares. Revista Novas Tecnologias na Educação, v. 11, n. 3, p. 1-10, 2013.

BERNARDES, W. C.; NOTARE, M. R. Os Objetos de Aprendizagem e o Desenvolvimento das Habilidades Espaciais. Revista Novas Tecnologias na Educação, v. 11, n. 3, p. 1-10, 2013.

BRITO, A. S.; LOPES, E. T. Professores de memória: construção da identidade docente dos primeiros formandos do curso de licenciatura em química de Itabaiana. Scientia Plena, v. 7, p. 1-16, 2011.

CLEMENT, J. J.; STEINBERG, M. S. step-wise Evolution of Mental Models of Eletric Circuits A learning-aloud case study.pdf. The Journal of the Learning Sciences, v. 11, n. 4, p. 389$452,2002$.

CLEMENT, J. J.; STEPHENS, A. L. Documenting the use of expert scientific reasoning process by high school physics students. Physics Education Research, v. 6, n. 2, p. 20122-1 - 20122-15, 2010.

FERREIRA, P. F. M.; JUSTI, R. DA S. Modelagem e o "Fazer Ciência."Química Nova, v. 1, n. 28, p. 32-36, 2008.

GALIAZZI, M. C.; MORAES, R. Análise Textual Discursiva. Ijuí: Editora Unijuí, 2011.

KABERMAN, Z.; DORI, Y. J. Question posing, inquiry, and modeling skills of chemistry students in the case-based computerized laboratory environment. International Journal of Science and Mathematics, v. 7, n. 3, p. 597-625, 2007. Springer.

LAUDAN, L. O Progresso e Seus Problemas: rumo a uma teoria do crescimento científico. Tradução Roberto Leal Ferreira. São Paulo: UNESP, Editora, 2011. 
MONAGHAN, J. M.; CLEMENT, J. J. Use of a computer simulation to develop mental simulations for understanding relative motion concepts. International Journal of Science Education, v. 21, n. 9, p. 921 - 944, 1999.

MOREIRA, M. A. Pesquisa em Educação em Ciências: Métodos Qualitativos. Universidad de Burgos, Espanha; Universidade Federal do Rio Grande do Sul, Brasil. Texto de Apoio ${ }^{\circ} 14$. Publicado em Actas del PIDEC. Anais... p.4:25-55, 2002.

POLANYI, M. Personal Knowledge: towards a post-critical Philosophy. Chicago: The University of Chicago Press, 1958.

RAUPP, D.; MOREIRA, M. A.; SERRANO, A. Desenvolvendo Habilidades Visuoespaciais: uso de software de construção de modelos moleculares no ensino de isomeria geométrica em química. Experiências em Ensino de Ciências, v. 4, n. 1, p. 65-78, 2009.

REBELLO, A. P.; RAMOS, M. G. Simulação Computacional e Maquetes na Aprendizagem de Circuitos Elétricos: um olhar sobre a sala de aula. Experiências em Ensino de C2iências, v. 4, n. 1, p. 23-33, 2009.

ROCHA, J. R.; SERRANO, A. Um Estudo de Caso Exploratório sobre a Internalização de Conceitos sobre Eletrostática: A influência da Hipercultura e Mediação Digital. Revista Novas Tecnologias na Educação, v. 11, n. 3, p. 1-10, 2013.

RODRIGUES, R. F. O Uso de Modelagens Representativas como Estratégia Didática no Ensino da Genética: um estudo de caso. Experiências em Ensino de CIências, v. 7, n. 2, p. 53-66, 2012.

SANTOS, F. M. T.; GRECA, I. M. Promovendo aprendizagem de conceitos e de representações pictóricas em Química com uma ferramenta de simulação computacional. Revista Electrónica de Enseñanza de las Ciencias, v. 4, n. 1, 2005.

SANTOS, H. F. O Conceito da Modelagem Molecular. Química Nova, , n. 4, p. 4-5, 2001.

SOUZA, B. C. DE. A Teoria da Mediação Cognitiva: Os impactos cognitivos da Hipercultura e da Mediação Digital, 2004. 282p, Tese (Doutorado em Psicologia) Centro de Filosofia e Ciências Humanas, Universidade Federal de Pernambuco, Recife. Disponível em: <http://www.liber.ufpe.br/teses/arquivo/20040617095205.pdf>. .

SOUZA, B. C.; SILVA, A. S.; SILVA, A. M.; ROAZZI, A.; SILVA, S. L. C. Putting the Cognitive Mediation Networks Theory to the test: Evaluation of a framework for understanding the digital age. Computers in Human Behavior, v. 28, n. 6, p. 2320-2330, 2012. Elsevier Ltd.

VAN-SOMEREN, M. W.; BARNARD, Y. F.; SANDBERG, J. A. C. The Think Aloud Method: a practical guide to modeling cognitive processes. London: Academic Press, 1994. 